\title{
Leiomyosarcoma of Uterine Cervix- A Case Report
}

\section{RENU THAMBI, SUBITHA K, LILLYKUTTY POTHEN, USHA POOTHIODE}

\section{ABSTRACT}

Primary sarcomas of cervix are rare lesions constituting $1 \%$ of the malignant tumors of cervix. Leiomyosarcoma of cervix is the commonest sarcoma of the cervix with only few cases reported in literature. We present a case of cervical leiomyosarcoma in a 49 years old female.
Primary sarcomas involving the cervix are rare constituting about $1 \%$; of which leiomyosarcoma is the commonest. Leiomyosarcoma is a malignant tumor showing smooth muscle differentiation with less than 100 cases reported in literature. Histogenesis of the tumor is thought to be from scattered smooth muscle cells in cervix.

\section{CASE HISTORY}

A 49 years old woman presented with bleeding per vaginum of 3 months duration. On examination patient was anaemic and there was no mass felt per abdomen. The cervix was enlarged and flushed with vault with a mass filling the vagina. Per vaginum the mass was bleeding on touch. There was no lymph node enlargement or evidence of secondaries noted. USG showed a heterogeneous lesion with calcification involving the cervix. CT scan of abdomen and pelvis showed a solid heterogeneously enhancing mass lesion involving the cervix. A biopsy taken from the mass showed poorly differentiated malignant cells with focal areas of spindling and moderate to marked pleomorphism. There was no area of epithelial differentiation in the biopsy. The neoplasm showed focal brownish black pigmentation which was negative on Masson Fontana stain. The possibilities considered were leiomyosarcoma, malignant melanoma and malignant mixed mullerian tumor. Patient further underwent total abdominal hysterectomy with debulking the tumor and bilateral salpingoophorectomy. Gross specimen showed circumferential enlargement of the cervix. On cut section the neoplasm involved the cervix diffusely and was gray white and firm and measured $5 \times 4.5 \times 4.5 \mathrm{~cm}$. It was extending into the lower uterine segment, paracervical tissue with a clearance of $2 \mathrm{~mm}$ and infiltrating the vaginal flap [Table/Fig-1A]. Body of uterus was free of the neoplasm. Microscopy showed the similar neoplasm as in the previous biopsy sections composed of spindly cells arranged in fascicles and sheets. Individual cells have spindle shaped nucleus with vesicular chromatin. Foci of necrosis, pleomorphism and atypical mitosis noted [Table/Fig-1B-C]. Immunohistochemistry showed positivity with smooth muscle actin (SMA) [inset Table/Fig-C]. and were negative for HMB 45 and CK (excluding melanoma and MMMT). Sections from uterus showed weakly proliferative endometrium and normal myometrium. Both ovaries and fallopian tubes showed no specific pathology. Omentum and abdominal lymph nodes were free of the neoplasm. Final diagnosis of malignant spindle cell neoplasmleiomyosarcoma of cervix was given. Post operatively the patient was advised pelvic radiation and chemotherapy. She disagreed to start the adjuvant therapy and was lost to follow up. After six months she was traced and brought back to our hospital where the adjuvant radiotherapy was initiated. The patient is now on treatment and she is doing well. No recurrence or metastasis documented.

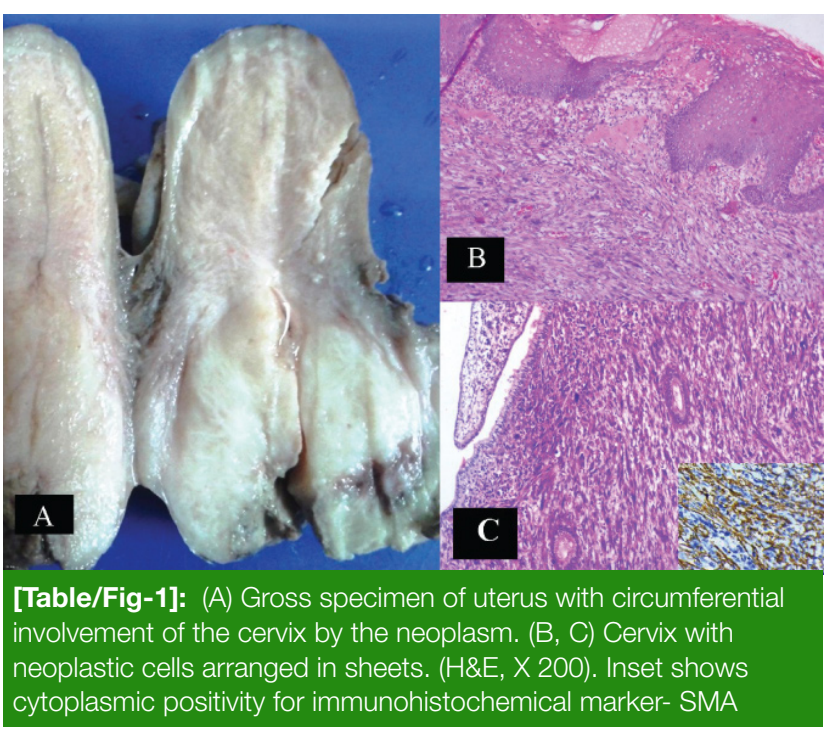

National Journal of Laboratory Medicine. 2016 Apr, Vol 5(2): 72-73 


\section{DISCUSSION}

Malignant mesenchymal tumors that can arise in the cervix include leiomyosarcoma, endocervical stromal sarcoma, embryonal rhabdomyosarcoma (botryoid type), alveolar soft part sarcoma, malignant schwannomas, and osteo sarcomas [1,2]. Primary cervical sarcomas are rare, of which the most common is leiomyosarcoma. Cervical leiomyosarcomas occur in the perimenopausal age group. Most common presentation is abnormal vaginal bleeding followed by urine retention [3,4]. Our patient presented with complaints of vaginal discharge and bleeding PV. Grossly, the tumors are large measuring about $12 \mathrm{~cm}$, poorly circumscribed masses that may protrude through the cervical canal or thicken and expand it circumferentially [2,3]. Primary leiomyosarcoma from uterine myometrium extending to the cervix has to be excluded [2]. Microscopically similar to the leiomyosarcoma of myometrium, a spectrum of morphologic types are seen, including the conventional, myxoid and epithelioid types. Owing to its rarity, criteria for malignancy followed are similar to that of tumors involving uterine corpus such as large size, pleomorphism, cellular atypia, necrosis and atypical mitosis [2,5-7]. Tumors with favourable and unfavourable outcome are defined. Large tumors, higher grade and stage, high proliferation index and in post menopausal age group are considered to have unfavourable outcome $[1,7,8]$. In our case there were cellular pleomorphism, atypical mitosis and necrosis, suggestive of malignancy. Immunohistochemistry with smooth muscle markers like smooth muscle actin, desmin and h-caldesmon are helpful. Malignant mixed mullerian tumor, sarcomatoid variant of squamous cell carcinoma and melanoma that can arise in cervix has to be excluded.

Not many studies have been published on the topic, but management should include abdominal hysterectomy with bilateral salpingo-oophorectomy. Pelvic lymphadenectomy is indicated in cases with lymph node metastasis. Adjuvant radiotherapy is used to prevent recurrence and chemotherapy is indicated in cases with metastasis. Chemotherapeutic regimes include VAC, doxorubicine and newer drugs like gemcitabine and docetaxel offer better results $[1,7,8]$. Combination of surgery, chemotherapy and radiotherapy is required for better outcome. Due to the lack of large series of cases of leiomyosarcoma of cervix the effect of different treatment modalities are not been well studied $[1-3,7,8]$.

\section{CONCLUSION}

We present this case due to the rarity of this lesion in cervix and the need to differentiate it from secondary involvement from leiomyosarcoma of uterine corpus. Prognostic predictors include tumor size, stage, grade, mitosis, age and menopausal status; are similar to uterine leiomyosarcomas. Combined modality of surgery, adjuvant radiation and chemotherapy should be used to achieve better survival.

\section{REFERENCES}

[1] Dhull AK, Kaushal V, Marwah N. The uncovered story of leiomyosarcoma of the cervix: a rare case report and review of literature. BMJ Case Rep. 2013,2013. doi: 10.1136/bcr-2013008616.

[2] Fadare $\mathrm{O}$. Uncommon sarcomas of the uterine cervix: a review of selected entities. Diagnostic pathology 2006; 1:30.

[3] Grover S, Abraham M, Mahajan MK. Leiomyosarcoma of the cervix. J Obstet Gynecol India. 2009; 59:364-66.

[4] Doshi B, Shetty S, Safaya A. Leiomyosarcoma of cervix. J Obstet Gynecol India. 2013; 63:211-12.

[5] Vellanki VS, Rao M, Sunkavalli CB, Chinamotu RN, Kaja S. A rare case of uterine leiomyosarcoma: a case report. Journal of Medical Case Reports. 2010;4:222.

[6] Subramaniam PM, Rekha PR, Thamilselvi R. An unusual clinical and morphological features of uterine leiomyosarcoma: immunohistochemistry solves the diagnostic pitfalls of uterine sarcomas. Journal of Clinical and Diagnostic Research. 2011 ;5(8): 1653-55.

[7] Bhatia V, Taksande R, Natekar A, Ali Z. Cervical leiomyosarcoma: a rare entity. South Afr J Gynaecol Oncol. 2015;7(2):64-66.

[8] Mehra R, Sidhu SPK, Pandey AK, Garg S. A rare case of leiomyosarcoma of cervix and challenges in its management: a case report and brief review of literature. Int J Res Med Sci. 2015;3:320-24
AUTHOR(S):
1. Dr. Renu Thambi
2. Dr. Subitha K
3. Dr. Lillykutty Pothen
4. Dr. Usha Poothiode

\section{PARTICULARS OF CONTRIBUTORS:}

1. Assistant Professor, Department of Pathology, Government Medical College, Kottayam, Kerala, India.

2. Assistant Professor, Department of Pathology, Government Medical College, Kottayam, Kerala, India.

3. Additional Professor, Department of Pathology, Government Medical College, Kottayam, Kerala, India.
4. Professor, Department of Pathology, Government Medical College, Kottayam, Kerala, India.

\section{NAME, ADDRESS, E-MAIL ID OF THE CORRESPONDING AUTHOR:}

Dr. Renu Thambi,

Assistant Professor, Department of Pathology, Government Medical College Kottayam, Kerala-686008, India.

E-mail: drrenu78@gmail.com

FINANCIAL OR OTHER COMPETING INTERESTS: None.

Date of Publishing: Apr 01, 2016 